\title{
ESTUDO MORFOLÓGICO DO RAMO SUPERFICIAL DO NERVO RADIAL
}

\author{
THE SUPERFICIAL BRANCH OF THE RADIAL NERVE: \\ A MORPHOLOGIC STUDY
}

\author{
Celso Ricardo Folberg ${ }^{1}$ \\ Heitor JR Ulson ${ }^{2}$ \\ Rodrigo Benedet Scheidt ${ }^{3}$
}

\section{RESUMO}

Objetivo: Estudar a anatomia e a histologia do ramo superficial do nervo radial (RSNR) no terço distal do antebraço e punho, através de dissecções em cadáveres. Métodos: Foram dissecadas 23 extremidades com auxílio de lupa de 3,5x para avaliar a distância do ponto de emergência do nervo até a apófise estilóide do rádio (AER); o número de ramos e fascículos em dois pontos distintos de seu percurso e outros achados anatômicos. Cortes histológicos do nervo e suas ramificações, cruzando o punho à mesma altura, foram realizados em 20 dissecções para contagem dos fascículos. Resultados: O ponto de emergência do RSNR entre os tendões do braquiorradial (BR) e o extensor radial longo do carpo (ERLC) foi encontrado em média a $8,65 \mathrm{~cm}$ da AER. Em seis casos (6/23) havia um tendão acessório do braquiorradial, com o RSNR emergindo entre este e o seu principal. A primeira ramificação teve origem em média 4,58 cm da AER. Em sete (7/23) casos havia um ramo cruzando o punho diretamente sobre a AER. A média do número de ramos cruzando o punho à altura da AER foi de 3,4. O número médio de fascículos do RSNR no seu ponto de emergência foi de 6,6 e em sua primeira ramificação, de quatro. Conclusão: Os achados anatômicos são semelhantes aos da literatura revisada, difundindo-se melhor conhecimento do RSNR. Há necessidade de extremo cuidado em procedimentos cirúrgicos da região estudada, principalmente naqueles ditos percutâneos que expõem as lesões do RSNR, pois em sete dos 23 casos o RSNR passou diretamente sobre a AER. Os autores recomendam a realização de pequenas incisões longitudinais, com dissecção romba e separação lateral de partes moles até o plano ósseo, antes de introduzir os fios de Kirschner em procedimentos minimamente invasivos.

Descritores - Nervo radial/anatomia; Osteossíntese; Cadáver

\section{ABSTRACT}

Objective: Study the morphology of the superficial branch of the radial nerve (SBRN) of the forearms and wrists of fresh adult human cadavers. Methods: Twenty three dissections were performed under 3.5x loupe magnification, histological sections of the nerve were obtained in 20 dissections for fascicle identification. Results: The SBRN emerged, in average, at $8.65 \mathrm{~cm}$ proximal to the radial styloid apophysis (RSA) between the Brachioradialis (BR) and Extensor Carpi Radialis Longus (ECRL) tendons. In 6/23 cases the SBRN emerged between an accessory $B R$ tendon and the main BR tendon. The first branch of the SBRN arose at an average of 4.58 $\mathrm{cm}$ proximal to the RSA. A branch running across the RSA was found in 7/23 cases. At that level, the average number of branches crossing the wrist was 3.4. A fascicle count of the nerve and its first branch showed an average of 6.6 and 4.0 fascicles, respectively. Conclusion: Our anatomical findings are similar to those in the revised literature and contribute towards a better knowledge of the SBRN. Great caution is required in surgical procedures such as percutaneous bone fixation of the distal 1/3 of the forearm and wrist and particularly, in those susceptible to SBRN injury, as in seven of the 23 cases the SRBN ran directly accross the RSA. The authors recommend performing small longitudinal incisions down to the subcutaneous tissue, separating the nerve branches by blunt soft tissue dissection, with a delicate haemostat, before introducing the Kirschner wires (minimally invasive procedures).

Keywords - Radial nerve/anatomy; Fracture fixation, internal; Cadaver

1 - Mestre em Cirurgia; Preceptor do Grupo de Cirurgia de Mão do Serviço de Ortopedia e Traumatologia do Hospital de Clínicas de Porto Alegre/RS

2 - Professor/Doutor do Departamento de Ortopedia e Traumatologia - Coordenador da Disciplina de Cirurgia da Mão - Faculdade de Ciências Médicas da Universidade Estadual de Campinas - UNICAMP/SP

3 - Médico Residente do $3^{\circ}$ ano do Serviço de Ortopedia e Traumatologia do Hospital de Clínicas de Porto Alegre/RS.

Trabalho realizado no Departamento de Cirurgia da Universidade Federal do Rio Grande do Sul - UFRGS, Porto Alegre, RS, e Departamento de Ortopedia e Traumatologia da UNICAMP, Campinas/SP, Brasil.

Correspondência: Rua Leopoldo Bier, 825 sala 305 - Porto Alegre - RS - Brasil - Cep 90620-100. E-mail: crfolberg@hotmail.com

Declaração: Não houve auxílio, de qualquer espécie, a esta pesquisa e não há conflitos de interesse dos autores em relação ao presente manuscrito, conforme Resolução $\mathrm{n}^{\circ} 1.595 / 2000$ do Conselho Federal de Medicina. 


\section{INTRODUÇÃO}

O ramo superficial do nervo radial (RSNR) deve ser encontrado e protegido nos acessos cirúrgicos ao rádio distal e articulações do punho. A sua descrição anatômica está citada em vários tratados e atlas de anatomia cirúrgica, porém, com pequenas diferenças entre eles. $\mathrm{Na}$ última década poucas descrições detalhadas do RSNR e seus ramos foram publicadas ${ }^{(1-6)}$. Porém, inúmeros casos de dor intratável causada por lesões neste nervo foram relatados ${ }^{(7-14)}$.

Pelo melhor conhecimento da topografia, de variações anatômicas e do número de fascículos do RSNR pode-se prevenir lesões cirúrgicas. O objetivo deste trabalho é estudar a anatomia e histologia do RSNR em seu trajeto na topografia da extremidade distal do rádio*.

\section{MÉTODOS}

Foram dissecados, com uso de lupa de aumento de $3.5 \mathrm{x}, 23$ antebraços e punhos de cadáveres frescos de adultos encaminhados para a seção de necropsia do Instituto Médico Legal (IML) de Porto Alegre. Não havia história e nem evidência de lesão aguda ou crônica nas extremidades dissecadas de 19 homens e de quatro mulheres.

Inicialmente uma agulha foi inserida percutaneamente marcando a apófise estilóide do rádio (AER) para medidas referenciais como demonstrado na figura 1.

A incisão foi iniciada distalmente, na base dorso-radial do polegar, indo em direção ao tubérculo de Lister (TL) estendendo-se proximalmente em "zigzag" sobre a face radial do antebraço em direção à prega flexora

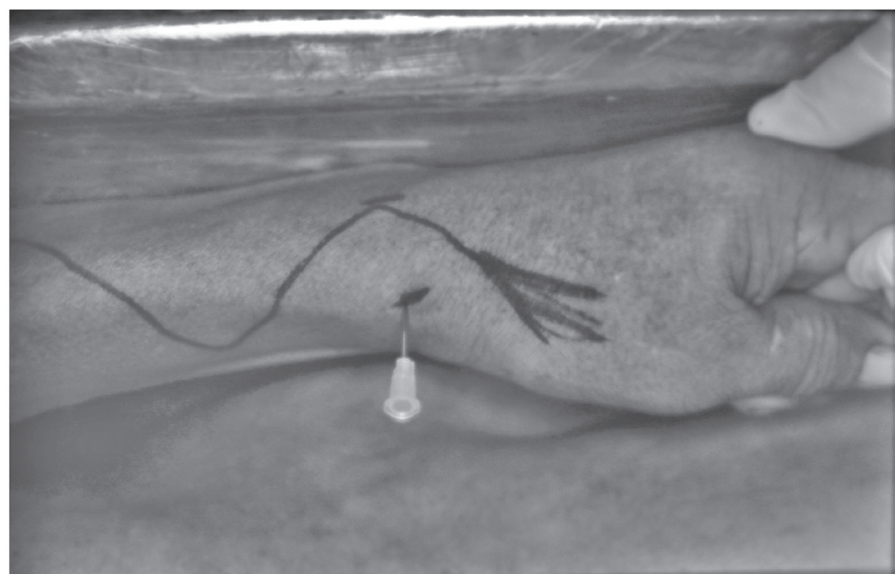

Figura 1 - Marcação da pele na extremidade para dissecção e colocação de agulha marcadora na AER. do cotovelo. O RSNR foi identificado junto aos tendões do músculo braquiorradial (BR) e do extensor radial longo do carpo (ERLC) no terço médio do antebraço e dissecado distalmente até o limite da incisão, com máximo cuidado para não alterar suas relações topográficas (Figura 2). Foi usado um paquímetro ( $\mathrm{N}^{\circ} 97031748$, Mitutoyo $^{\circledR}$, Brasil) para medir a distância desde a AER ao ponto de emergência do RSNR, junto aos tendões do BR e ERLC no terço médio do antebraço (Figura 3), e a distância da AER à origem do primeiro ramo do RSNR (Figura 4). O comprimento do rádio foi medido na posição neutra de prono-supinação, indo da sua cabeça ao processo estilóide, usando-se uma régua metálica milimetrada com $30 \mathrm{~cm}$ de comprimento.

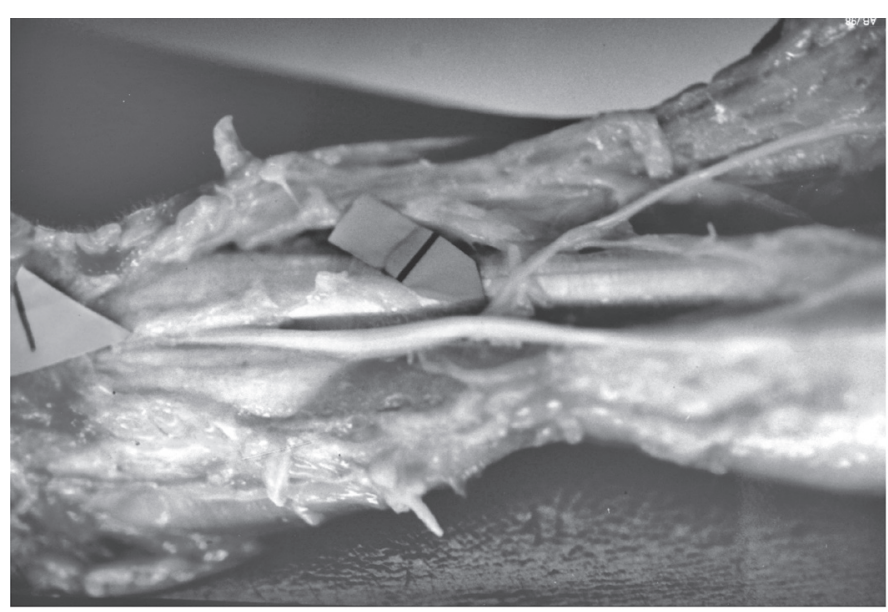

Figura 2 - Seta à esquerda: emergência do RSNR entre os tendões do BR e ERLC. Seta à direita: primeiro ramo do RSNR.

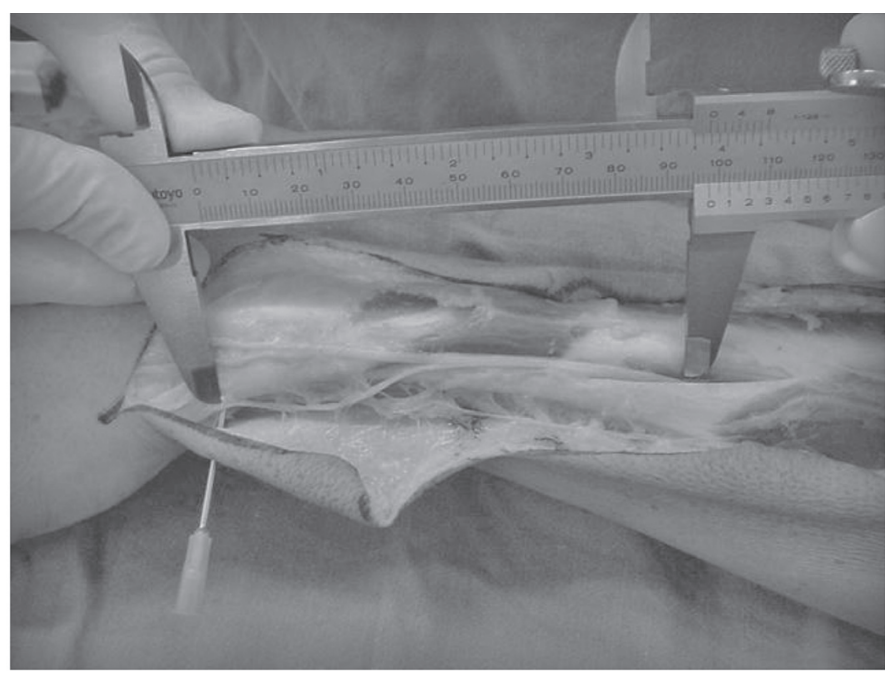

Figura 3 - Medida da distância entre o ponto de emergência do RSNR e a AER com paquímetro.

* Folberg, Celso Ricardo. Estudo anatômico-histológico do ramo superficial do nervo radial no terço distal do antebraço e punho. Campinas, SP: Universidade Estadual de Campinas, UNICAMP. Faculdade de Ciências Médicas; 2002. 


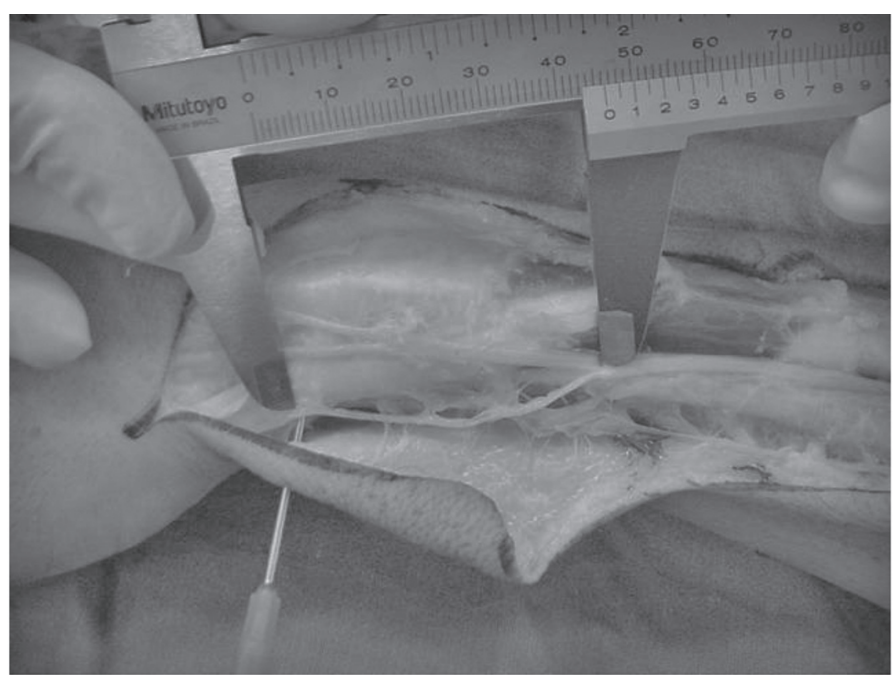

Figura 4 - Medida da distância entre a AER à origem do primeiro ramo do RSNR.

Os ramos dorsal e volar que cruzam o punho próximo à $\mathrm{AER}$ e o ramo mais próximo ao TL, também foram identificados e mensuradas as referidas distâncias (Figura 5). Também foram estudados o número de tendões acessórios do BR e o número de ramos do RSNR cruzando a articulação do punho na altura da AER.

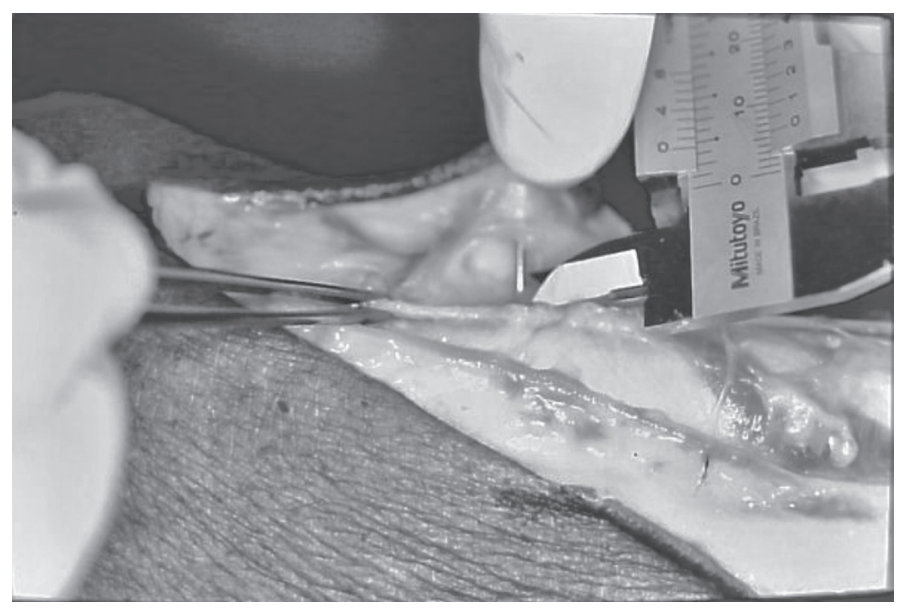

Figura 5 - Medida da distância entre o ramo do RSNR mais próximo à $A E R$ e a mesma (marcada com agulha) com paquímetro.

Foram realizados cortes transversais do nervo para análise histológica em dois locais diferentes de seu trajeto: em sua emergência e no local onde todos os ramos cruzam o punho na altura da AER. Este procedimento foi realizado em 20 casos, iniciando-se no quarto antebraço dissecado. Os cortes foram identificados e colocados em solução de formalina (figuras 6 e 7) sendo, em seguida, numerados de um a seis, de radial para ulnar, de acordo com a sua proximidade à AER e corados com hematoxilina-eosina para análise dos fascículos. Este trabalho é resultado de uma tese de mestrado avaliada e aprovada pelos critérios ético-legais da Faculdade de Medicina da UNICAMP.

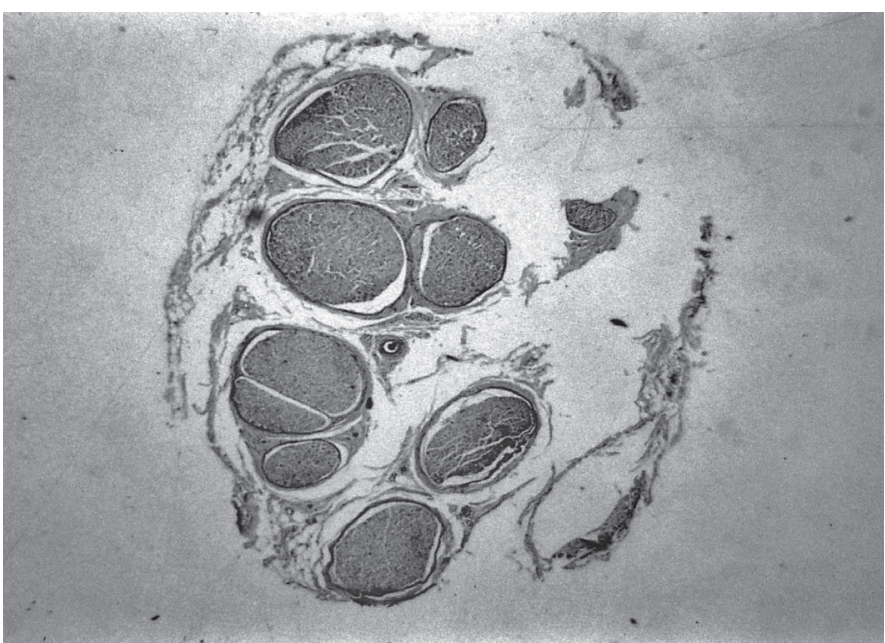

Figura 6 - Corte histológico do RSNR no seu ponto de emergência com a visualização de oito fascículos (coloração hematoxilina-eosina).

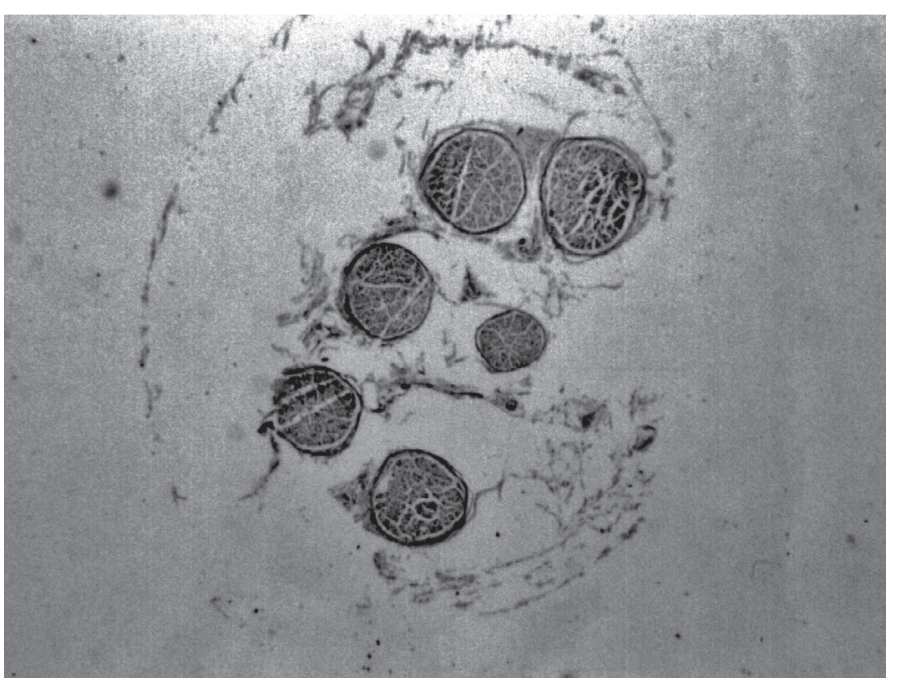

Figura 7 - Corte histológico de um ramo do RSNR à altura do punho com a visualização de seis fascículos (coloração hematoxilina-eosina).

\section{RESULTADOS}

Apresentamos os resultados de 23 dissecções anatômicas e análise histológica em 20 extremidades de cortes transversais para estudar a distribuição fascicular.

O comprimento médio do osso rádio nos 23 casos foi de $23 \mathrm{~cm}(19,5 \mathrm{~cm}$ a 25,9 cm, desvio padrão $\mathrm{DP}=1,53)$. O RSNR foi identificado no terço médio do antebraço 
entre os tendões do braquiorradial (BR) e extensor radial longo do carpo (ERLC) na maioria das dissecções, exceto em seis casos (6/23), em que foi identificado um tendão acessório do BR, ocasião em que o ramo surgia entre os dois tendões do BR (Figura 8). O ponto de emergência entre os dois tendões foi em média de 8,65 $\mathrm{cm}$ proximais à $\operatorname{AER}(5,5 \mathrm{~cm}$ a $11,2 \mathrm{~cm} ; \mathrm{DP}=1,36 \mathrm{~cm})$, esta distância correspondendo a $37 \%$ do comprimento do rádio.

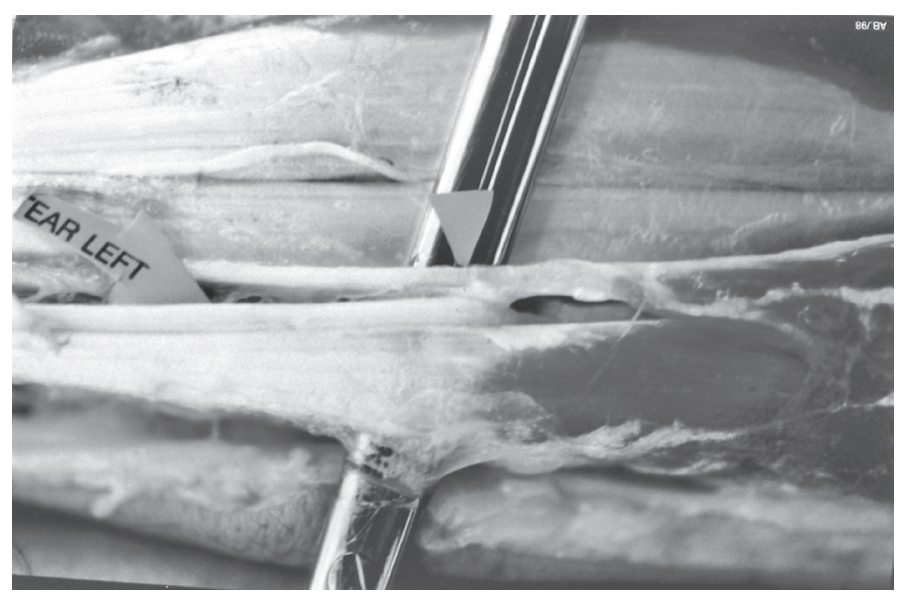

Figura 8 - RSNR (seta horizontal à esquerda) emergindo entre os tendões acessório (seta vertical à direita) e principal do BR.

A primeira grande ramificação foi identificada quase sempre no lado radial, originando-se em média a $4.5 \mathrm{~cm}$ proximais à $\operatorname{AER}(2,0 \mathrm{a} 7,9 \mathrm{~cm} ; \mathrm{DP}=1.39)$. Em apenas uma ocasião foi identificado um fino ramo no lado ulnar com origem a $0,3 \mathrm{~cm}$ proximal à saída da primeira ramificação do lado radial. Em sete casos (7/23), um ramo foi identificado passando diretamente sobre a AER (Figura 9). O ramo mais próximo ficou em média a $0,38 \mathrm{~cm}$ da AER ( 0 a $1,2 \mathrm{~cm}$; DP $=0,34)$. Em apenas um caso $(1 / 23)$, foi identificado um ramo passando diretamente sobre o tubérculo de Lister (TL), e em nenhum, foi identificado algum ramo no lado ulnar do TL.

$\mathrm{O}$ número médio de ramos do RSNR cruzando o punho no nível da AER foi de 3,4 ( 2 a 6; DP=1.34).

Em duas extremidades foi observada comunicação com ramos do nervo cutâneo lateral do antebraço.

No local do ponto de emergência foram encontrados em média 6,6 fascículos do RSNR (2 a 13; DP=2,87) (Figura 6). Em sua primeira ramificação e ao nível do punho, em todas as extremidades estudadas, foram encontrados, em média, quatro fascículos (dois a sete; $\mathrm{DP}=1,64$ ); na segunda ramificação, direcionando-se de

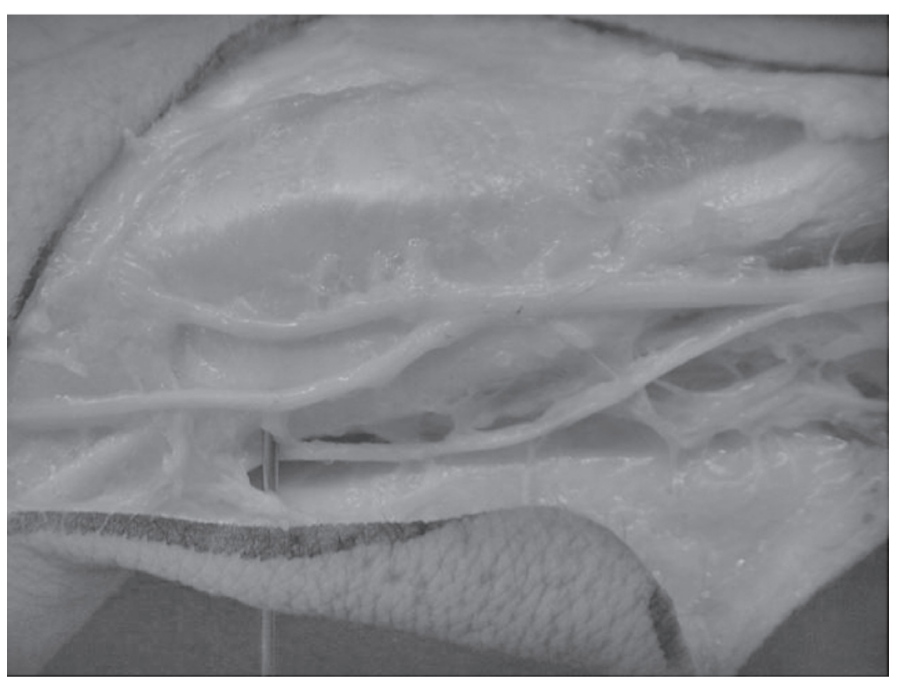

Figura 9 - Fotografia demonstrando o RSNR cruzando exatamente sobre a AER a qual aparece marcada com agulha.

radial para ulnar, em 20 extremidades encontrou-se uma média de 4,5 fascículos (2 a 11; DP=2,67); na terceira, encontrada em 12 extremidades (12/20), a média foi 3,5 (1 a 9; $\mathrm{DP}=2,46)$; na quarta ramificação, encontrada em sete das 20 extremidades (7/20), a média foi de 3,1 fascículos (1 a 5; DP=1,58); e na quinta, encontrada em cinco casos $(5 / 20)$, a média foi de três e na sexta ramificação, encontrada em duas extremidades (2/20), a média foi de quatro fascículos (2 a $6 ; \mathrm{DP}=2,82$ ).

A acurácia dos cálculos e dos resultados foi confirmada pelo teste de Komolgorov e Smirnov ( $p<0,05$ em todos os resultados).

\section{DISCUSSÃO}

O punho e o terço distal do antebraço são sítios freqüentes de procedimentos cirúrgicos quer percutâneos ou abertos, onde podem ocorrer lesões ao RSNR e geralmente com resultados indesejáveis e às vezes até desastrosos.

Estudos apresentam detalhes da anatomia do RSNR com resultados obtidos por meio de 20 dissecções, sendo que Kuhlmann et al apresentaram 50 extremidades dissecadas $^{(15)}$. Outro trabalho enfatiza detalhes anatômicos com aplicações nos portais artroscópicos ${ }^{(16)}$. Estudos do primeiro compartimento extensor chamam atenção para o uso de fios de Kirschner na tabaqueira anatômica ${ }^{(17-19)}$. A inervação cutânea do dorso da mão com suas implicações clínicas, as características anatômicas do RSNR, e o fato de o mesmo estar relacionado com síndromes compressivas no antebraço, realçam a importância deste ramo do nervo radial ${ }^{(5,6,14)}$. 
Encontramos a emergência do RSNR em média a $8,64 \mathrm{~cm}$, proximais à $\mathrm{AER}$, entre os tendões do $\mathrm{BR}$ e ERLC, valor próximo daqueles encontrados por outros autores com médias entre 7,8 a $9,0 \mathrm{~cm}^{(1-3,5,15,20)}$. Lanzetta et al reforçam clinicamente estes achados ao encontrar o sinal de Tinel na síndrome compressiva do RSNR a $8,8 \mathrm{~cm}$ proximais à $A E R$, levando em conta que a compressão se dá na emergência deste ramo entre os tendões do BR e ERLC ${ }^{(21)}$. Em relação à primeira ramificação do RSNR, a distância média da AER foi de $4,58 \mathrm{~cm}$, a qual foi a mesma encontrada por Leroux et al e inferior àquelas encontradas por Ikiz et $\mathrm{al}^{(5)}$, Gonzalez et $\mathrm{al}^{(17)}$, Abrams et al ${ }^{(1)}$, Auerba-

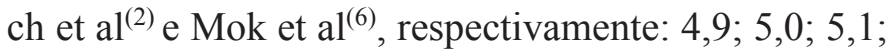
5,3 e $5,5 \mathrm{~cm}$.

Na presente série não foi encontrado o RSNR emergindo através do tendão principal do $\mathrm{BR}$, como referido em duas dissecções por Abrams et al ${ }^{(1)}$. Entretanto, em seis dissecções (6/23), descrevemos o RSNR emergindo entre um tendão do BR acessório e o seu tendão principal. Este achado também havia sido descrito por outro autor em 5\% dos 143 pacientes submetidos a cirurgia neste local ${ }^{(20)}$. Em estudo anterior, os mesmos autores relataram a presença de um tendão acessório do $\mathrm{BR}$ em 6\% das 150 dissecções, porém, o RSNR localizou-se entre os tendões acessório e principal do BR em apenas $3,3 \%$ dos $\operatorname{casos}^{(22)}$. Devido ao fato de termos encontrado possível freqüência maior de tendões acessórios do $\mathrm{BR}$, em relação às séries de Turkof et al, justifica-se a incidência de tal variação anatômica ${ }^{(20,22)}$. O aproveitamento desses tendões acessórios pode ser importante em transferências tendinosas variadas.

Os tratados e atlas tradicionais de anatomia demonstram normalmente um ou dois ramos do RSNR cruzando o punho na altura da AER, fato parcialmente confirmado pelas 50 dissecções realizadas por Kuhlman et al que identificaram dois ramos: superficial lateral e superficial medial. No presente estudo encontramos uma variação de duas a seis ramificações neste nível. Auerbach et al chegaram a encontrar um caso com 10 ramos, mas a média foi de 5,8, número este próximo ao encontrado por Leroux et al que foi de 5.1 ramos em média do RSNR ao nível da $\operatorname{AER}^{(2,6)}$.

Acreditamos que a maior relevância clínica deste trabalho está em demonstrar que em sete casos (7/23), pelo menos um ramo do RSNR cruzou o punho exatamente sobre a AER (figura 9), local de eleição para referência na inserção de fios de Kirschner quando re- alizados procedimentos percutâneos para as fraturas da extremidade distal do rádio. Em estudo experimental sobre a introdução percutânea de fios de Kirschner evidenciou-se lesão ou deslocamento do RSNR em $32 \%$ dos $\operatorname{casos}^{(23)}$. Existem muitos relatos na literatura de lesão do RSNR no nível do punho ${ }^{(7-14,16,18,19,23)}$. O conhecimento das variações anatômicas deste nervo e a realização de pequenas incisões longitudinais com dissecção romba e separação lateral de partes moles até o plano ósseo, pode contribuir para a prevenção deste tipo de lesão. Seria preferível denominá-los de procedimentos minimamente invasivos e assim descrevê-los, pois o termo percutâneo sugere introduções às cegas, conseqüentemente com maior risco de lesão nervosa.

Não encontramos na literatura outro trabalho com citação do número de fascículos por ramificação do RSNR no terço distal do antebraço. Nossos resultados apresentam uma curva de distribuição normal de acordo com o teste de Kolmogorov-Smirnov. Em nenhum caso deste estudo o número de fascículos do nervo principal foi igual à soma dos de suas ramificações, ou seja, ao longo de um nervo existem divisões, anastomoses e migrações de fascículos que justificam nossos achados.

$\mathrm{O}$ número de fascículos encontrados no local de emergência do RSNR apresentou variações consideráveis, de dois a 13 fascículos com média de 6,6 fascículos e $\mathrm{DP}=2,87$. Não encontramos outro estudo na literatura para compararmos este dado, portanto, até o momento, podemos considerar com 6.6 o número médio de fascículos do RSNR.

Acreditamos que estudos mais detalhados da anatomia do RSNR serão de extrema importância para prevenir, diagnosticar e tratar as lesões deste nervo.

\section{CONCLUSÃO}

Os achados anatômicos são semelhantes aos da literatura revisada, difundindo-se melhor conhecimento do RSNR. Há necessidade de extremo cuidado em procedimentos cirúrgicos da região estudada, principalmente naqueles ditos percutâneos que expõem as lesões do RSNR, pois em sete dos 23 casos o RSNR passou diretamente sobre a AER. Os autores recomendam a realização de pequenas incisões longitudinais, com dissecção romba e separação lateral de partes moles até o plano ósseo, antes de introduzir os fios de Kirschner em procedimentos minimamente invasivos. 


\section{REFERÊNCIAS}

1. Abrams RA, Brown RA, Botte MJ. The superficial branch of the radial nerve: an anatomic study with surgical implications. J Hand Surg Am.1992;17(6):1037-41.

2. Auerbach DM, Collins ED, Kunkle KL, Monsanto EH. The radial sensory nerve: an anatomic study. Clin Orthop Relat Res. 1994;(308):241-9.

3. Leroux M, Harris P, Fowles JV. An anatomic study of the superficial radial nerve and its clinical implications. Ann Chir. 1998;52(8):736-43.

4. Ndiaye A, Diop M, Dia A, Seye SI, Sow ML. The sensitive branch of the radial nerve at the inferior third of the forearm and at the wrist. Clinical applications. Bull Assoc Anat (Nancy). 1996;80(249):27-30.

5. Ikiz ZA, Üçerler H. Anatomic characteristics and clinical importance of the superficial branch of the radial nerve. Surg Radiol Anat. 2004;26(6):453-58.

6. Mok D, Nikolis A, Harris PG. The cutaneous innervation of the Dorsal Hand: detailed anatomy with clinical implications. J Hand Surg Am. 2006;31:565-74.

7. Arons MS. De Quervain's release in working women: a report of failures, complications and associated diagnoses. J Hand Surg Am. 1987; 12:540-4.

8. Braidwood AS. Superficial radial neuropathy. J Bone Joint Surg Br. 1975;57:380-3.

9. Linscheid RL. Injuries to the radial nerve at the wrist. Arch Surg. 1965;91:942-7.

10. Louis DS, Greene TL, Noellert RC. Complications of carpal tunnel surgery. J Neurosurg. 1985;62(3):352-6.

11. Sanders RA, Keppel FR, Waldrop JI. External fixation of distal radius fractures: results and complications. J Hand Surg Am. 1991;16:385-91.

12. Thrush DN, Belsole R. Radial nerve injury after routine peripheral vein cannulation. J Clin Anesth. 1995;7:160-2.
13. Singh S, Trikha P, Twyman R. Superficial radial nerve damage due to Kirschner wiring of the radius. Injury. 2005;36:330-2.

14. Dellon AL, Mackinnon SE. Susceptibility of the superficial sensory branch of the radial nervo to form painful neuromas. J Hand Surg Br. 1984;9:42-5.

15. Kuhlmann JN, Guerin-Surville H. The superficial branch of the radial nerve. Topographical location. Systematization. Bull Assoc Anat (Nancy). 1985;669(207): 331-41.

16. Abrams RA, Petersen M, Botte MJ. Arthroscopic portals of the wrist: an anatomic study. J Hand Surg Am. 1994;19:940-4.

17. Gonzalez MH, Sohlberg R, Brown A, Weinzweig N. The first dorsal extensor compartment: an anatomic study. J Hand Surg Am. 1995;20:657-60.

18. Leão L. De Quervain's disease: a clinical and anatomical study. J Bone Joint Surg Am. 1958;40:1063-70.

19. Steinberg BD, Plancher KD, Idler RS. Percutaneous Kirschner wire fixation through the snuff box: an anatomic study. J Hand Surg Am. 1995;20:57-62.

20. Turkof E, Puig S, Choi MS, Zoch G, Dellon AL. The radial sensory nerve entrapped between the two slips of a split brachioradialis tendon: a rare aspect of Wartenberg's syndrome. J Hand Surg Am. 1995;20:676-8.

21. Lanzetta $M$, Foucher $G$. Entrapment of the superficial branch of the radial nerve. A report of 52 cases. Int Orthop. 1993;17:342-6.

22. Turkof E, Puig S, Choi MS, Schilhan R, Millesi H, Firbas W. Superficial branch of the radial nerve emerging between two slips of a split brachioradialis muscle tendon: a variation of possible clinical relevance. Acta Anat (Basel). 1994;150(3):232-4.

23. Hochwald NL, Levine R, Tornetta P. The risks of Kirschner-wire placement in the radius: a comparision of techniques. J Hand Surg Am. 1997;22:580-4. 